\title{
A Few Observations of the Perturbations in the Phase of the Low-Frequency Ground Wave ${ }^{1,2}$
}

\author{
J. M. Ross and J. E. Kirch
}

(December 5, 1960; revised March 6, 1961)

\begin{abstract}
The effects of anomaly-producing terrain features on the phase of the low frequency ground wave were measured in a nearly idealized environment to confirm theoretical predictions: Results for an isolated butte gave good correlation with the theoretical model. In most cases the mean value of the phase perturbation approached zero.
\end{abstract}

\section{Introduction}

Low frequencies have been used for radio navigation for some time with excellent results obtained over water and in the air. However, in use on the ground, disturbances caused by cultural and terrain features, and changes in conductivity cause errors in position.

Many tests [Bateman, 1950, 1951; Schneider, 1952 ; Pressey, 1956] of various systems have been conducted and considerable information is available in unpublished reports for these systems on the type of cultural and terrain features that cause propagation disturbances. However, little information is found of sufficiently general nature to be applied or extended to other systems. In addition, propagational theory is usually developed only for idealized cases, so that measurements are necessary to determine the limitations of idealized cases or mathematical models.

\section{Measurement Program}

A comprehensive program was conducted in the desert near Florence, Arizona to obtain a quantitative look at the order of magnitude and exact nature of these disturbances. The particular area was selected because it contained several anomaly producers in isolation. It contains mountains, buildings, power lines and telephones, all isolated from each other by relatively large areas of flat desert (see fig. 1).

For example, the area of measurement was restricted so that the range to all transmitters was less than $80 \mathrm{~km}$ to minimize sky wave reflection and effects of earth curvature. Measurements were made with a double-heterodyne, CW, hyperbolic, phase-measuring equipment operating at 480 and $450 \mathrm{kc} / \mathrm{s}$.

Further limiting of variables was achieved by taking readings along a single line of position in

$\overline{1}$ Contribution from Motorola, Inc., Military Electronics Division, Western Center, Scottsdale, Arizona.

2 This paper was presented originally at the Seventh Annual East Coast Conference on Aeronautical and Navigation Electronics, October 24-26, 1960, Conference on Aeronautical and Navigation Electronics, October 24-26, 1960, of Ships under Contract NObsr 72739 . lieu of using position fixes. Even with the single line of position, particular care was taken to position the receivers so as to measure the effect of phase changes from only one transmitter.

The principle of operation is common to several different systems operating on different frequencies. It has been described in the literature and will not be covered here [International Hydrographic Office, 1956 and Kirch, 1959]. It was found that equipment developed for tactical applications could be modified simply to serve as scientific measuring instruments. Three measuring stations were used; a fixed monitor to assure equipment stability, a ground van, and a helicopter. Measuring points were tied by first-order triangulation and trilateration to the U.S. Coast and Geodetic survey of the area. All data were recorded by photographic techniques and reduced by an IBM 650 computer. Reported here is error in line of position data as determined by the phase of a $480-\mathrm{kc} / \mathrm{s}$ carrier. The errors are given in meters and degrees of phase with respect to distance expressed either in meters or wavelengths.

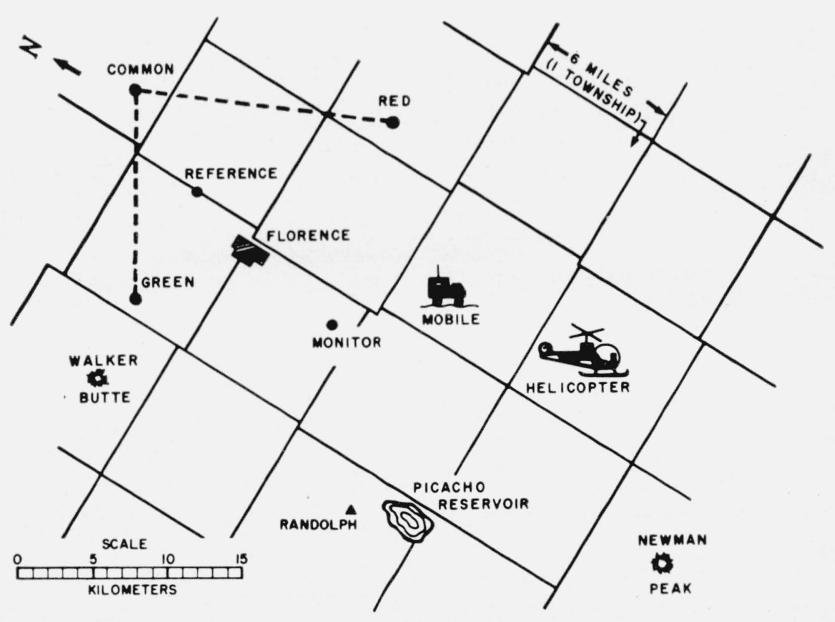

Figure 1. Map of test area. 


\subsection{Obstacle-Free Area}

Measurements were made in a relatively obstaclefree area. A triangulation station near Randolph, Arizona was used as an obstacle-free area because the nearest mountain is $24 \mathrm{~km}$ and the entire area is surrounded by relatively flat desert thought to be homogeneous.

Bremmer [1949] derived a rigorous solution suitable for numerical computation for the propagation of the low-frequency ground wave over a homogeneous spherical earth. At short ranges, the series approximations involved converge very slowly. However, use can be made of the plane earth solution due to Norton [1941]. Both solutions show the field to consist of the primary factor and the secondary factor. The primary factor, or undisturbed field, propagates with a velocity determined by the index of refraction of the atmosphere at the earth's surface. The secondary factor includes all disturbances to the field due to the presence of the earth and the antenna (near field effect).

At the ranges used in this research, the only secondary effect is that due to retardation of the wave by the earth acting as a lossy boundary, since measurements were made at ranges large enough that the near field had no effect. Neglecting near field effect then, the phase correction due to ground absorption increases monotonically with distance.

Since calculation of the secondary factor is quite complicated and because the conductivity of the earth is difficult to measure, only the primary factor was taken into account in the data reduction computation.

Measurements were taken along a line extending $914 \mathrm{~m}$ south, $305 \mathrm{~m}$ east, and $305 \mathrm{~m}$ north of the monument. Results appear in figure 2. No significant effect of terrain clearance was noted. The mean error of $2.1 \mathrm{deg}$ is less than that expected had the secondary factor been taken into account. A small cyclic variation occurred with a period of a half wavelength. This variation is thought to be caused by a mountain at $24 \mathrm{~km}$. A telephone line

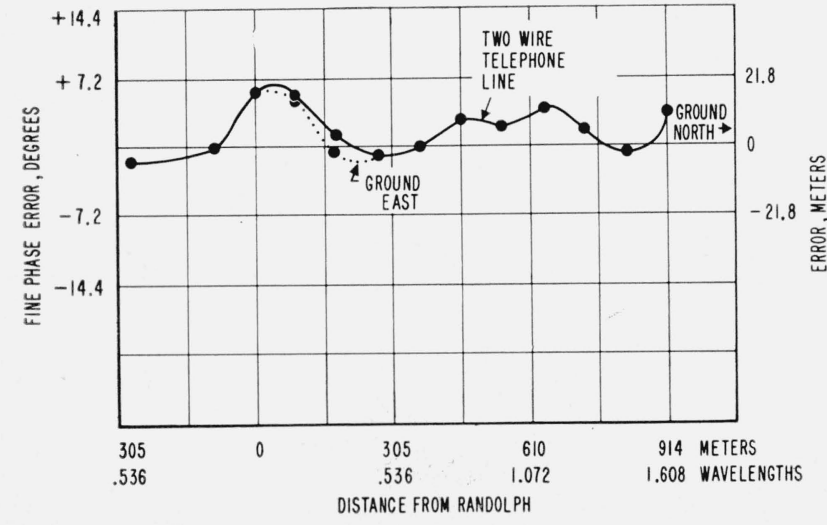

Figure 2. Phase perturbations when anomalies are not apparent. in the immediate vicinity caused no noticeable effect, since the error was identical for travel parallel to and perpendicular to the line. The small cyclic error can be eliminated by integrating over several wavelengths.

\subsection{Effect of a Mountain}

Of particular interest is the case of an isolated mountain in the transmission path. At high frequencies the effect of mountains obstructing the transmission path can be treated by methods of physical optics; but another approach must be used when the obstacles are no longer large compared to a wavelength. Such is the case considered by Wait and Murphy [1956 and 1958] for the effect of the amplitude and phase of a plane wave scattered by a semi-cylindrical boss. The boss of finite conductivity is placed on an infinite plane that is perfectly conducting. A source is assumed to be located many wavelengths from the boss; hence, the incident wave can be considered to be a plane wave. Attenuation of the incident wave is neglected.

On the surface of the plane the incident wave is

$$
H^{i n c}=H_{o} e^{i k x}
$$

where $H_{o}$ is the amplitude of the magnetic field and $k$ the wave number. The scattered field due to a boss of radius $r$ and conductivity $\sigma$ is

$$
H^{\mathrm{sec}}=-H_{o} \sum_{m=0}^{\infty} K_{m}(k r, \sigma) H_{m}^{(2)}(k x) .
$$

$K_{m}(k r, \sigma)$ is a series of constants dependent on $k r$ and $\sigma$ for the index $m$. $H_{m}^{(2)}(k x)$ is a Hankel function of the second kind of order $m$. The "phase lag" of the resultant field is

$$
k x-\arg \left[\frac{H^{\mathrm{sec}}+H^{i n c}}{H_{o}}\right] .
$$

The amplitude and phase of the total field (i.e., incident field plus the scattered field) at various points around the boss, are plotted in the report by Wait and Murphy [1958].

On the near side of the boss, the variation in the phase is essentially a damped sinusoid decreasing in a direction away from the boss. On the far side, the error decreases monotonically in an essentially exponential manner away from the boss.

Unfortunately, mountains of the type and shape of the model do not often exist in nature, especially in isolation. The mountain chosen (Walker Butte), however, did answer the required description adequately enough to enable test of the theory. This butte rises $500 \mathrm{ft}$ above the relatively flat surrounding desert and is approximately hemispherical in shape. Its relation to the transmitters is shown in figure 3. Data was taken for a distance of $3,000 \mathrm{ft}$ starting at a distance $2,665 \mathrm{ft}$ from the peak. Surveying was done using accurately known points. Aerail readings were taken at terrain clearances 
of 30,150 , and $500 \mathrm{ft}$ with no significant difference in results obtained between ground and aerial readings.

Figure 4 shows plots of phase error versus distance from the butte as actually measured, and for the mathematical model taken from Wait and Murphy [1958]. As the figure indicates, experimental results agree remarkably well with the theory. The calculated correlation index is 0.5 .

At very great distances from a long ridge, the asymptotic expression for $H_{m}^{(2)}(k x)$ can be used.

$$
\lim _{k x \rightarrow \infty} H_{m}^{(2)}(k x)=\sqrt{\frac{2}{\pi k x}}(e)^{i\left(k x-\frac{\pi}{4}-m \frac{\pi}{2}\right) .}
$$

This equation would indicate that the attenuation of the scattered field at great distances is proportional to the square root of distance. At these frequencies, the cyclic error noted at Randolph, Ariz., could be attributed to the mountain $24 \mathrm{~km}$ away.

\subsection{Effect of a Critically Placed Power Line}

The perturbation of phase due to vertical parasitic reradiators has been covered theoretically and experimentally [Bateman, 1950, 1951; Hufford, 1950]. A literature search failed to uncover any theoretical treatment of the effects of power lines on phase and amplitude of the low-frequency ground wave [Reynolds, 1953]. Considerable data was taken in the vicinity of power lines and telephone lines. In the general case the effects were local, with most telephone lines having negligible effect.

It has been suggested that power lines might be considered a Beverage antenna. ${ }^{3}$ The theoretical effects of the line are, to a great extent, determined by terminations, capacity and other electrical properties which could not be determined or controlled for a line in service.

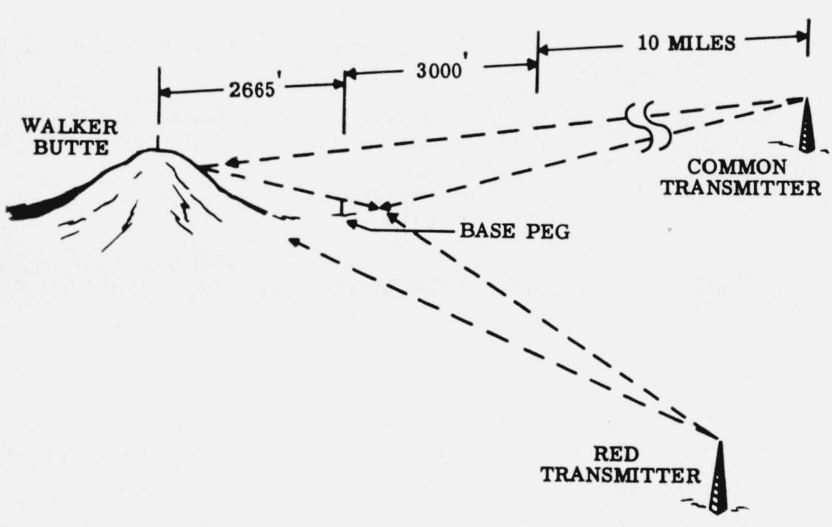

Figure 3. Sketch of Walker Butte.

${ }^{3}$ Private communication: R. F. Linfield, R. H. Doherty, and G. Hefley, Evaluation of propagation aspects of cytac system.

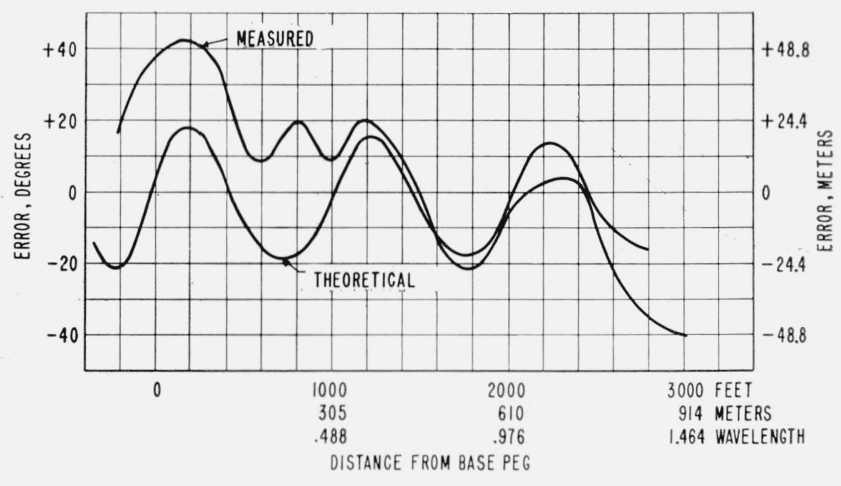

Figure 4. Comparison of measurements at Walker Butte with, the predicted.

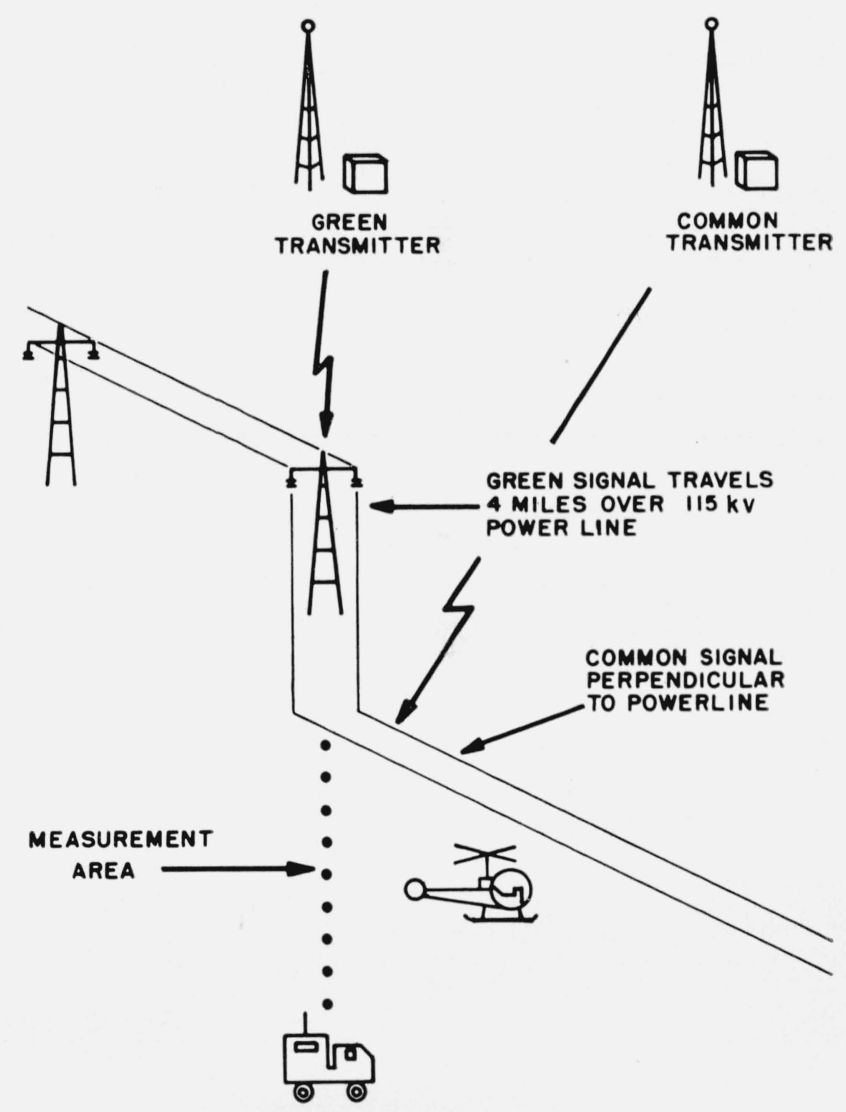

Figure 5. Relation of power line to test transmitters.

To measure this effect, a unique transmitter location was chosen. The green transmitter was deliberately positioned so that the signal would travel for $61 \frac{1}{2} \mathrm{~km}$ along a $115-\mathrm{kv}$ line (see fig. 5). The line turns at an angle of $30^{\circ}$, making it possible to take measurements along the extension of the line. The common transmitter signal crosses the line at right angles. 
Figure 6 shows how the relative error varies with distance away from the power line. The test grid system at this location was not tied into the U.S.C.G.S. survey, so that absolute errors cannot be reported precisely. From Land Office survey data and other readings taken in the vicinity, there is reason to believe that the magnitude of the absolute error is two or three times that of the relative error. At a triangulation station, $4 \frac{1}{2} \mathrm{~km}$ along the extension, the absolute error reduced to zero when the secondary factor calculated for earth conductivity of 6 millimhos/m was taken into account.

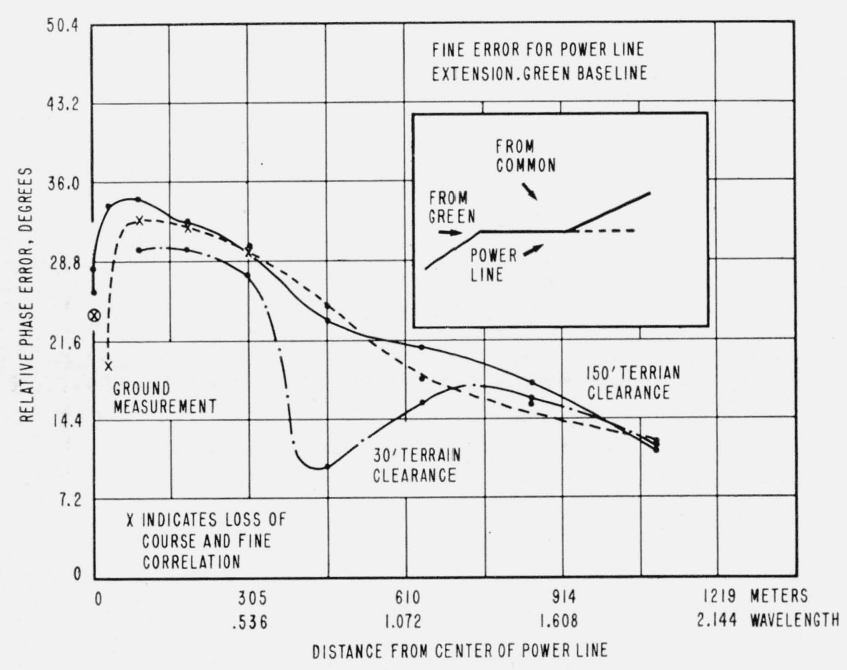

Figure 6. Phase perturbations for a power line.

(Paper 65D4-143)

\section{Conclusions}

Phase perturbations of the low-frequency ground wave occur in areas where the cause is not apparent, but the mean value of the perturbations approaches zero. Measurements tend to confirm the model used for the perturbations due to an isolated mountain. Tests made near a power line would indicate that more investigation of the Beverage antenna as a model are in order.

\section{References}

Bateman, R., Florman, E. F., and Tait, A., A source of error in radio phase measuring systems, Proc. IRE, Vol. 38, No. 6, 612-614 (June 1950).

Bateman, R., Florman, E. F., and Tait, A., Discussion on a "Source of error in radio phase measuring systems", Proc. IRE, Vol. 39, No. 4, 436-438 (April 1951).

Bremmer, H., Terrestrial radio waves, (New York, Elsevier, 1949).

International Hydrographic Bureau, Radio aids to maritime navigation and hydrography, International Hydrographic Bureau, S. P. No. 39 (MONACO, 1956).

Kirch, James E., A low-frequency navigation system with a simple airborne display, Proc. of National Aeronautical Electronics Conf., Dayton, Ohio (May 1959).

Norton, K. A., The calculation of ground-wave field intensity over a finitely conducting spherical earth, Proc. IRE, Vol. 29, No. 12, 623-639 (Dec. 1941).

Reynolds, L. G., An examination of some site and transmission path errors of the Decca Navigator System when used over land. Proc. IEE, Vol. 100, Pt III, No. 63, 29-35 (January 1953).

Schneider, A. B., Phase variations with range of the groundwave signal from $\mathrm{cw}$ transmitters in the $70-130 \mathrm{kc} / \mathrm{s}$ band, J. Brit. IRE, Vol. 12, 181-194 (March 1952).

Wait, James R., and A. Murphy, Influence of a ridge on the low frequency ground wave, J. Research NBS, Radio Propagation 58D, No. 1, 1-5 (Jan. 1957).

Wait, James R., and A. Murphy, Further studies of the influence of a ridge on the low frequency ground wave, J. Research NBS, Radio Propagation, 61D, No. 1, 57-60 (July 1958).

Pressey, B. G., G. E. Ashwell, and C. S. Fowler, Change of phase with distance of a low-frequency ground wave propagated across a coastline. Proc. IEE, Vol. 103, Pt B No 10, 527-534 (July 1956). 